\title{
A note on zero-sum 5-flows in regular graphs
}

\author{
S. Akbari ${ }^{a, b}$, N. Ghareghani ${ }^{d, b}$, G. B. Khosrovshahi ${ }^{b, e}$, S. Zare ${ }^{c}$ \\ ${ }^{a}$ Department of Mathematical Sciences, Sharif University of Technology \\ ${ }^{b}$ School of Mathematics, Institute for Research in Fundamental Sciences (IPM) \\ P.O. Box 19395-5746 \\ ${ }^{c}$ Department of Mathematical Sciences, Amirkabir University of Technology \\ ${ }^{d}$ Department of Engineering Science, College of Engineering, University of Tehran \\ P.O. Box 11165-4563, Tehran, Iran \\ e Department of Mathematics, University of Tehran $* \dagger \ddagger$
}

Submitted: Oct 13, 2010; Accepted: Apr 7, 2012; Published: Apr 16, 2012

\begin{abstract}
Let $G$ be a graph. A zero-sum flow of $G$ is an assignment of non-zero real numbers to the edges of $G$ such that the sum of the values of all edges incident with each vertex is zero. Let $k$ be a natural number. A zero-sum $k$-flow is a flow with values from the set $\{ \pm 1, \ldots, \pm(k-1)\}$. It has been conjectured that every $r$-regular graph, $r \geqslant 3$, admits a zero-sum 5 -flow. In this paper we provide an affirmative answer to this conjecture, except for $r=5$.
\end{abstract}

\section{Introduction}

Nowhere-zero flows on graphs were introduced by Tutte [8] in 1949 and since then have been extensively studied by many authors. A great deal of research in the area has been motivated by Tutte's 5-Flow Conjecture which states that every 2-edge connected graph can have its edges directed and labeled by integers from $\{1,2,3,4\}$ in such a way that Kirchhoff's current law is satisfied at each vertex. In 1983, Bouchet [4] generalized this concept to bidirected graphs. A bidirected graph $G$ is a graph with vertex set $V(G)$ and edge set $E(G)$ such that each edge is oriented as one of the four possibilities:

*E-mail addresses: s_akbari@sharif.edu (S. Akbari), ghareghani@ipm.ir (N. Ghareghani), rezagbk@ipm.ir (G.B. Khosrovshahi), sa_zare_f@yahoo.com (S. Zare).

${ }^{\dagger}$ Keywords: Zero-sum flow, regular graph.

†AMS (2010) Subject classification: 05C21, 05C22.

${ }^{\S}$ Corresponding author: G.B. Khosrovshahi. 
Let $G$ be a bidirected graph. For every $v \in V(G)$, the set of all edges with tails (respectively, heads) at $v$ is denoted by $E^{+}(v)$ (respectively, $E^{-}(v)$ ). The function $f: E(G) \longrightarrow \mathbb{R}$ is a bidirected flow of $G$ if for every $v \in V(G)$, we have

$$
\sum_{e \in E^{+}(v)} f(e)=\sum_{e \in E^{-}(v)} f(e)
$$

If $f$ takes its values from the set $\{ \pm 1, \ldots, \pm(k-1)\}$, then it is called a nowhere-zero bidirected $k$-flow.

Bouchet proposed the following interesting conjecture.

Bouchet's Conjecture. [4, 9] Every bidirected graph that has a nowhere-zero bidirected flow admits a nowhere-zero bidirected 6-flow.

Bouchet showed that his conjecture is true if 6 is replaced by 216. Then Zyka [10] reduced 216 to 30. Also, DeVos [5] proved Bouchet's Conjecture with 6 replaced by 12 .

Let $G$ be a graph. A $k$-regular graph is a graph where each vertex is of degree $k$. A zero-sum flow of $G$ is an assignment of non-zero real numbers to the edges of $G$ such that the sum of the values of all edges incident with each vertex is zero. Let $k$ be a natural number. A zero-sum $k$-flow is a flow with values from the set $\{ \pm 1, \ldots, \pm(k-1)\}$. The following conjecture was posed on the zero-sum flows in graphs.

Zero-Sum Conjecture (ZSC). [1] If $G$ is a graph with a zero-sum flow, then $G$ admits a zero-sum 6-flow.

The following theorem shows the relation between Bouchet's Conjecture and ZSC.

Theorem 1. [2] Bouchet's Conjecture and ZSC are equivalent.

The following conjecture is a stronger version of ZSC for regular graphs.

Conjecture A. [2] Every $r$-regular graph $(r \geqslant 3)$ admits a zero-sum 5-flow.

Motivated by Bouchet's Conjecture and along with Theorem 1 we focused our attention to establish the Conjecture A. In the next section, except for the case $r=5$, we prove Conjecture A. The following two results show the validity of this conjecture for some special cases.

Theorem 2. [1] Let $r$ be an even integer with $r \geqslant 4$. Then every $r$-regular graph has a zero-sum 3-flow.

Theorem 3. [2] Let $G$ be an $r$-regular graph. If $r$ is divisible by 3 , then $G$ has a zero-sum 5-flow. 
Remark. There are some regular graphs with no zero-sum 4-flow. To see this consider the graph given in Figure 1. Assume, to the contrary, that this graph has a zero-sum 4 -flow. Since the sum of the values of all three edges incident with a vertex is zero, not all can be odd, so -2 or 2 should appear on (exactly) one edge incident to the vertex. On the other hand two numbers with absolute value 2 cannot appear in the neighborhood of a vertex. So the edges of $G$ with values \pm 2 form a perfect matching. But by a celebrated Theorem of Tutte [3, p.76], $G$ has no perfect matching, a contradiction.

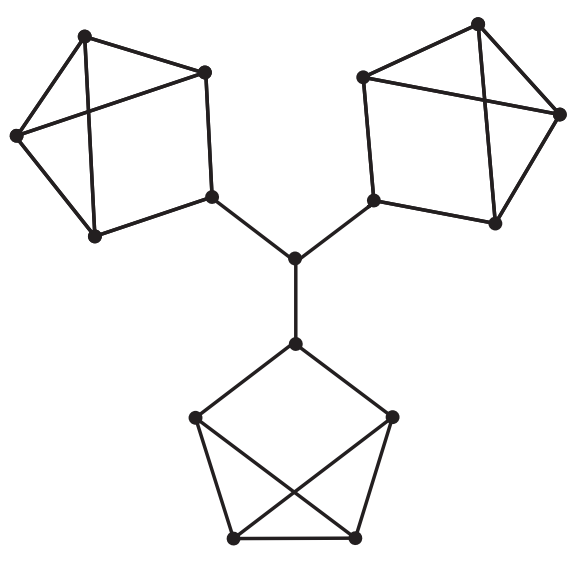

Figure 1: A 3-regular graph with no zero-sum 4-flow

\section{The Main Result}

In this section we prove that every $r$-regular graph, $r \geqslant 3, r \neq 5$, admits a zero-sum 5-flow. Before establishing our main result we need some notations and definitions.

A factor of a graph is a spanning subgraph. A $k$-factor is a factor which is $k$-regular. In particular a 2-factor is a disjoint union of cycles that cover all the vertices. Let $G$ be a graph with vertex set $V(G)$ and edge set $E(G)$. A $k$-factorization of $G$ is a partition of the edges of $G$ into disjoint $k$-factors. For integers $a$ and $b, 1 \leqslant a \leqslant b$, an $[a, b]$-factor of $G$ is defined to be a factor $F$ of $G$ such that $a \leqslant d_{F}(v) \leqslant b$, for every $v \in V(G)$. For any vertex $v \in V(G)$, let $N_{G}(v)=\{u \in V(G) \mid u v \in E(G)\}$.

Below we state two known theorems about the factorization of graphs.

Theorem 4. [7] Every 2k-regular multigraph admits a 2-factorization.

Theorem 5. [6] Let $r \geqslant 3$ be an odd integer and let $k$ be an integer such that $1 \leqslant k \leqslant \frac{2 r}{3}$. Then every $r$-regular graph has a $[k-1, k]$-factor each component of which is regular.

Lemma 6. Let $G$ be an $r$-regular graph. Then for every even integer $q, 2 r \leqslant q \leqslant 4 r$, there exists a function $f: E(G) \rightarrow\{2,3,4\}$ such that for every $u \in V(G), \sum_{v \in N_{G}(u)} f(u v)=q$. 
Proof. For every edge $e=u v$, we add a new edge $e^{\prime}=u v$ to the graph $G$ and call the resulting graph $G^{\prime}$. Clearly, $G^{\prime}$ is a $2 r$-regular multigraph. By Theorem $4, G^{\prime}$ admits a 2 -factorization with 2 -factors $F_{1}, \ldots, F_{r}$. Now, for every $e \in F_{i}, 1 \leqslant i \leqslant r$, we define a function $g: E\left(G^{\prime}\right) \rightarrow\{1,2\}$ as follows:

$$
g(e)= \begin{cases}2, & 1 \leqslant i \leqslant \frac{q-2 r}{2} \\ 1, & \frac{q-2 r}{2}<i .\end{cases}
$$

Therefore, for each $u \in V\left(G^{\prime}\right), \sum_{v \in N_{G^{\prime}}(u)} g(u v)=q$. Now, define a function $f$ : $E(G) \rightarrow\{2,3,4\}$ such that for every $e=u v \in E(G), f(e)=g(e)+g\left(e^{\prime}\right)$, where $e^{\prime}=u v$ in $G^{\prime}$. Then for every $u \in V(G), \sum_{v \in N_{G}(u)} f(u v)=q$, as desired.

Now, we are in a position to prove our main theorem.

Theorem 7. Let $r \geqslant 3$ and $r \neq 5$. Then every $r$-regular graph has a zero-sum 5-flow.

Proof. If $r=3$, then by Theorem 3, the assertion holds. First we prove the theorem for $r=7$. Let $G$ be a 7-regular graph. Then, by Theorem $5, G$ is a disjoint union of a 3-regular graph $H_{1}$ and a 4-regular graph $H_{2}$. By Theorem $4, H_{2}$ can be decomposed into two 2-factors $H_{2}^{\prime}$ and $H_{2}^{\prime \prime}$. Assign 1 and 2 to all edges of $H_{2}^{\prime}$ and $H_{2}^{\prime \prime}$, respectively. By Lemma 6 , there exists a function $f: E\left(H_{1}\right) \rightarrow\{2,3,4\}$ such that for every $u \in V\left(H_{1}\right)$, $\sum_{v \in N_{H_{1}}(u)} f(u v)=8$. Now, assign -2 to every edge in $E(G) \backslash E(H)$ and we are done.

Now, let $r \geqslant 9$ be an odd integer. By Theorem 5 , for every $k, k \leqslant \frac{2 r}{3}, G$ has a $[k-1, k]$ factor whose components are regular. Let $k=\left\lfloor\frac{2 r}{3}\right\rfloor, k^{\prime}=r-k$, and $H$ be a $[k-1, k]$-factor of $G$ such that $H_{1}$ is the union of the $(k-1)$-regular components of $H$ and $H_{2}=H \backslash H_{1}$. It can be easily checked that $k \leqslant 2 k^{\prime} \leqslant 2 k-4$. Hence by Lemma 6 , there exists a function $f: E\left(H_{1}\right) \longrightarrow\{2,3,4\}$ such that for every $u \in V\left(H_{1}\right), \sum_{v \in N_{H_{1}}(u)} f(u v)=4 k^{\prime}+4$. Also by Lemma 6 , there exists a function $f: E\left(H_{2}\right) \longrightarrow\{2,3,4\}$ such that for every $v \in V\left(H_{2}\right), \sum_{v \in N_{H_{2}}(u)} f(u v)=4 k^{\prime}$. Finally assign -4 to every edge of $E(G) \backslash E(H)$. Now, by Theorem 2 the proof is complete.

Acknowledgements. The authors are indebted to the School of Mathematics, Institute for Research in Fundamental Sciences (IPM) for the support. The research of the first author and the second author were in part supported by grants from IPM (No. 90050212) and (No. 88050042), respectively.

\section{References}

[1] S. Akbari, G. B. Khosrovshahi, A. Mahmoody and N. Ghareghani, On zero-sum 6-flows of graphs, Linear Algebra and its Applications 430 (2009), 3047-3052.

[2] S. Akbari, A. Daemi, O. Hatami, A. Javanmard and A. Mehrabian, Zero-sum flows in regular graphs, Graphs Combin. 26 (2010), 603-615.

[3] J.A. Bondy, U.S.R. Murty, Graph Theory with Applications, North Holland, New York, 1976. 
[4] A. Bouchet, Nowhere-zero integral flows on a bidirected graph, J. Combin. Theory, Ser. B 34 (1983), 279-292.

[5] M. DeVos, Flows in bidirected graphs, preprint.

[6] M. Kano, Factors of regular graphs, J. Combin. Theory Ser. B 41 (1986), 27-36.

[7] J. Petersen, Die theorie der regulären graphs, Acta Math. (15) (1891), 193-220.

[8] W.T. Tutte, On the imbedding of linear graphs in surfaces, Proc. London Math. Soc. Ser 251 (1949), 464-483.

[9] R. Xu and C.Q. Zhang, On flows in bidirected graphs, Discrete Math. (2005), No. 299, 335-343.

[10] O. Zyka, Nowhere-zero 30-flow on bidirected graphs, Charles University, Praha, 1987, KAM-DIMATIA Series 87-26. 